médecine/sciences $1985: 1: 35^{8-62}$

\title{
La maladie des chaînes alpha
}

C'est la plus fréquente des maladies des chaînes lourdes. Elle touche le système de l'IgA sécrétoire, habituellement le tractus digestif. A une hyperplasie lymphoplasmocytaire, parfois réversible après antibiotiques, succède un lymphome malin. La structure très particulière des molécules d'immunoglobulines qui caractérisent ces maladies pose des problèmes qui commencent à être résolus par la biologie moléculaire.

\section{Maxime Seligmann \\ Professeur d'immunologie à la fa- culté de médecine Lariboi- sière-Saint-Louis, Université Paris VII. Directeur de l'Unité 108 de l'Inserm.}

\section{REFERENCES}

I. Seligmann M, Danon F, Hurez D, Mihaesco E, Preud'hommc JL. Alpha chain disease: a new immunoglobulin abnormality. Science $1968 ;$ I 62 : I 396-7.

2. Seligmann $\mathbf{M}$, Mihaesco $E$, Hurez D, Mihaesco C, Preud'homme JL, Rambaud JC. Immunochemical studies in four cases of alpha chain disease. 7 Clin Invest $1969 ; 48: 2374-89$.

3. Radl J. Light chain typing of immunoglobulins in small samples of biological materials. Immunology 1970; 19 : 1 37-49.

4. Doe WF, Danon F, Seligmann M. Immunodiagnosis of alpha chain disease. Clin Exp Immunol 1979; 36 : 1 89-97.

5. Rambaud JC, Galian A, Danon F, et al. Alpha chain disease without qualitative serum IgA abnormality. Report of two cases, including a " nonsecrctory "form. Cancer I983; 5 I : 686-93.

6. Seligmann M, Mihaesco E, Preud'homme JL, Danon F, Brouet JC. Heavy chain diseases: current findings and concepts. Immunol Rev I979; $48: 145-67$.

\section{ADRESSE}

M. Seligmann : Inserm U Io8, hôpital SaintLouis, 75475 Paris Cedex Io. es maladies des chaînes lourdes (MCL) sont des syndromes immunoprolifératifs observés chez l'homme et caractérisés par la production d'immunoglobulines (Ig) particulières, par l'absence de chaînes légères et par la présence de chaînes lourdes anormalement courtes, délétées (figure थ). Des MCL ont été décrites pour chacune des trois principales classes de chaines lourdes humaines $\gamma, \alpha$ et $\mu$; celle des chaînes $\alpha \quad(\mathrm{MCL} \alpha)$, décrite en I 968 [1] est la plus fréquente.

Toutes les MCL soulèvent des problèmes biologiques très intéressants concernant la structure de l'Ig pathologique qui les caractérise (localisation précise de la région de la chaîne lourde qui est délétée 't nature de la séquence amino-terminale) et le (ou les) mécanisme(s) de génétique cellulaire responsable(s) de la synthèse de cette chaîne lourde délétée, ainsi que, dans la plupart des cas, de l'absence de production de chaînes légères. La MCL $\alpha$ représente en outre un modèle privilégié pour comprendre la pathogénie des lymphomes humains. Son épidémiologie très particulière implique en effet le rôle, dans la genèse de la prolifération lymphoide, de certains microorganismes induisant des stimulations antigéniques; son évolution comporte un stade initial d'infiltration lymphoplasmocytaire parfois réversible sous antibiothérapie orale et un stade terminal caractérisé par un lymphome de haute malignité.
Compte tenu de leur définition, le diagnostic des MCL repose entièrement sur le laboratoire.

\section{Diagnostic biologique de la maladie}

Le diagnostic de MCL $\alpha$ consiste en général à démontrer, par des méthodes immunochimiques, la présence dans les sérum du malade d'une population de molécules d'IgA dépourvues de chaînes légères. Les techniques utilisées en routine ne permettent pas, en règle générale, de démontrer que les chaînes lourdes $\alpha$ sont délétées.

Dans la moitié des cas environ, on ne voit pas d'anomalie nette sur le tracé électrophorétique des protéines sériques, du fait du taux relativement faible de la protéine pathologique. Lorsqu'il existe une bande anormale sur le tracé électrophorétique, elle est le plus souvent hétérogène. L'analyse immunoélectrophorétique du sérum est un élément essentiel du diagnostic, montrant la présence d'une protéine qui réagit avec les immunsérums anti- $\alpha$ mais ne réagit pas avec les immunsérums anti- $\kappa$ et anti- $\lambda$. Cette protéine peut parfois passer inaperçue lorsqu'on utilise pour l'examen un antisérum polyvalent. L'absence de précipitation avec des sérums anti-chaînes légères n'est pas un critère suffisant pour affirmer le diagnostic puisque ceci peut également s'observer avec certaines globulines myélomateuses comportant des chaînes légères, en particulier $\operatorname{Ig} \mathrm{A} \lambda$. 
Deux méthodes spécifiques et sensibles permettent d'affirmer la présence d'une protéine de MCL $\alpha$. L'une est l'immunoélectrophorèse conventionnelle mais utilisant des antisérums anti-Ig A sélectionnés qui, après absorption par les chaînes légères, contiennent, outre les anticorps anti-chaînes lourdes, une quantité appréciable d'anticorps réagissant avec les déterminants antigéniques conformationnels de la région $\mathrm{Fab}$, nécessitant la présence simultanée de la chaîne lourde et de la chaîne légère [2]. Lorsqu'on utilise de tels immunsérums, les molécules d'IgA normales (présentes dans le sérum ou apportées par l'adjonction de sérum normal au sérum du malade) forment un éperon sur la ligne de précipitation de la protéine de MCL. L'autre méthode est l'immunosélection [3] avec incorporation dans la gélose d'immunsérums anti-chaînes légères $\kappa$ et $\lambda$ sélectionnés de manière à contenir des anticorps de forte affinité, capables de précipiter toutes les molécules d'Ig entières, monoclonales ou polyclonales. La technique d'immunosélection la plus sûre et la plus simple est celle qui est combinée à l'immunoélectrophorèse. Une étude comparative de ces deux méthodes[4] a montré que la seconde est un peu plus sensible que la première et que sa lecture est plus aisée pour un observateur modérément expérimenté. En cas d'immunosélection combinée à l'immunoélectrophorèse, l'utilisation d'un immunsérum anti-Fab contenant des anticorps qui réagissent avec les déterminants conformationnels (au lieu d'immunsérums anti- $\kappa$ et anti$\lambda$ ) augmente la sensibilité et la sécurité de la technique pour le diagnostic de MCL $\alpha[4]$.

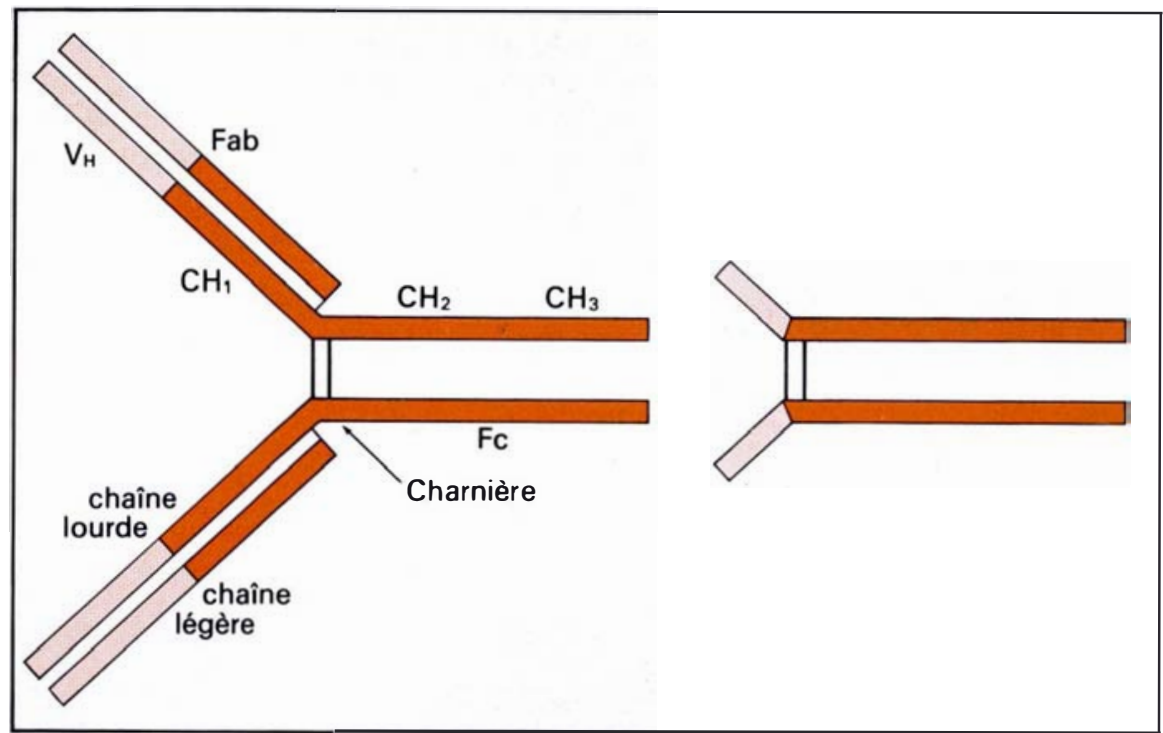

Figure 1. Représentation schématique de la structure d'une molécule d'immunoglobuline (appartenant à la sous-classe IgAI) comparée à la structure d'une protéine de maladie des chaînes lourdes $\alpha$. La molécule d'immunoglobuline comporte une paire de chaînes légères ( $\kappa$ ou $\lambda$ ) et une paire de chaînes lourdes (ici $\left.I_{I}\right)$. Chaque chaîne comprend une partie aminoterminale variable (en rose) et une partie carboxyterminale constante (en rouge). La chaîne lourde est constituée de quatre régions (ou domaines) d'environ IIO acides aminés : $V_{H}$ (région variable des chaînes lourdes); $\mathrm{CHI}$ ( $I^{e r}$ domaine constant); $\mathrm{CH}_{2}$ (2 ${ }^{e}$ domaine constant); $\mathrm{CH}_{3}$ (3 $^{e}$ domaine constant). Entre $\mathrm{CHI}$ et $\mathrm{CH}_{2}$, se trouve la région charnière avec les ponts disulfures reliant les deux chaînes lourdes. Chacune de ces quatre régions de la partie constante est codée par un exon distinct. La scission de la molécule par la papaïne produit deux fragments Fab comprenant une chaîne légère et les régions $V_{H}$ et $C H_{I}$ d'une chaîne lourde et un fragment $\mathrm{Fc}$ comprenant les régions charnière, $\mathrm{CH}_{2}$ et $\mathrm{CH}_{3}$ des deux chaînes lourdes. La protéine de maladie des chaînes lourdes ne comporte pas de chaîne légère. Elle correspond à un dimère de chaînes lourdes anormalement courtes constituées le plus souvent d'une partie de la région variable $V_{H}$, de la région charnière et des domaines $\mathrm{CH}_{2}$ et $\mathrm{CH}_{3}$.

$m / s n^{0} 7$ novembre 85

Les mêmes méthodes immunochimiques permettent de démontrer la présence de la protéine de MCL $\alpha$ dans le suc intestinal, parfois même en l'absence de protéine pathologique décelable dans le sérum. La protéine de MCL $\alpha$ est absente, ou présente en quantité minime, dans les urines.

Toutes les protéines de MCL $\alpha$ qui ont été typées appartiennent à la sous-classe $\alpha_{1}$. L'absence de MCL $\alpha_{2}$ est peut-être liée au relatif éloignement sur le chromosome I4 des exons codant pour les sous-classes $\alpha_{1}$ et $\alpha_{2}$. Cette constatation nous avait aussi fait émettre l'hypothèse d'une dégradation rapide des protéines de la sous-classe $\alpha_{2}$ après leur synthèse ou leur sécrétion. Il s'agirait alors de formes "non-sécrétantes " de MCL $\alpha$ (analogues aux myélomes non-sécrétants) qui pourraient rendre compte des cas dans lesquels le contexte clinicopathologique est analogue à celui d'une forme digestive de MCL $\alpha$ (classiquement dit : "lymphome méditerranéen ") mais où on ne décèle la protéine pathologique, ni dans le sérum, ni dans le liquide jéjunal. Longtemps suspectée, cette éventualité a pu être récemment démontrée [5]. Le diagnostic de MCL $\alpha$ au niveau de la prolifération cellulaire, fort utile en pareil cas, repose sur des études en immunofluorescence ou par immunopéroxydase qui montrent la présence de chaînes $\alpha$ dépourvues de chaînes légères. Les expériences de biosynthèse permettent de démontrer la production de chaines $\alpha$ anormalement courtes.

\section{Structure de la protéine pathologique}

Les molécules d'Ig produites dans les MCL sont des chaînes lourdes délétées. Les principales données sur la structure des protéines de MCL $\alpha$ et de MCL en génćral [6] (figure 2, voir p. suivante) peuvent être ainsi résumées. L'endroit où, après la délétion, il y a reprise d'une séquence normale de la région constante d'une chaîne lourde de même classe (ou sous-classe) se situe pratiquement toujours au début d'un domaine. Pour les 4 protéines de MCL $\propto$ qui ont été étudiées par 


\section{RÉFÉRENCES}

7. Wolfenstein-Todel C, Mihaesco E, Frangione B. "Alpha chain discase " protein DEF : interna deletion of a human immunoglobulin $\mathrm{A}$ heavy chain. Proc Natl Acad Sci USA r 974; 7 I : 974-8

8. Barnikol-Watanabe S, Mihaesco E, Mihaesco C, Barnikol HU, Hilschmann N. The primary structure of $\mu$ chain disease protein BOT. Peculiar amino-acid sequence of the $\mathrm{N}$-terminal 42 positions. Hoppe-Seylers Z Physiol Chem 1984; 365: $105-18$

9. Alexander A, Steinmetz M, Barritault D, et al. $\gamma$-heavy chain disease in man : cDNA sequence supports partial gene deletion model. Proc $\mathrm{Natl}$ Acad Sci USA 1982; $79: 3260-4$.

ı. Guglielmi P, Bakshi A, Mihaesco E, Brouet JC, Waldmann TA, Korsmeyer SJ. DNA deletion in human gamma heavy chain disease. Clin Res $1984 ; 32: 348$ (abstr).

I I. Bakshi A, Siebenlist U, Guglielmi P, et al. Molecular analysis of human mu heavy chain disease. Clin Res 1984; $32: 342$ (abstr.).

12. Preud'homme JL, Brouet JC, Seligmann M. Cellular immunoglobulins in human $\gamma$ and $\alpha$ heavy chain diseases. Clin Exp Immunol I 979; $37: 283-91$.

13. Cowan NJ, Secher DS, Milstein C. Intracellular immunoglobulin chain synthesis in nonsecreting variants of a mouse myeloma : detection of inactive light chain messenger RNA. J Mol Biol 1974; 90:691-701.

14. Dackowski W, Morrison SL. Two heavy chain disease proteins with different genomic deletions demonstrate that non-expressed heavy chain genes contain methylated bases. Proc Natl Acad Sci USA 198I; $78: 7091-5$.

15. Rambaud JC, Seligmann M. Alpha chain disease. Clin Gastroenterol 1976; $5: 34$ I-58.

I6. Galian AM, Lecestre J, Scotto J, Bognel C, Matuchansky C, Rambaud JC. Pathological study of alpha-chain disease, with special emphasis on evolution. Cancer 1977; $39: 208$ I-IOI.
E. Mihaesco dans notre laboratoire, la séquence normale reprend au résidu 222, au début de la région charnière. C'est aussi le cas pour de nombreuses protéines de MCL $\gamma$ alors que, pour les trois protéines de MCL $\mu$ dont la séquence a été étudiée, la reprise d'une séquence normale s'effectue au début de $\mathrm{CH}_{\mathrm{I}}$ pour l'une, de $\mathrm{CH}_{2}$ pour la seconde et de $\mathrm{CH}_{3}$ pour la troisième $(f i-$ gure I). La délétion porte donc en général sur deux domaines $\left(V_{H}\right.$, en partie ou en totalité, et $\mathrm{CHI}$ ) ou, plus rarement, sur trois domaines. A partir du point de réinitiation, la séquence est celle de la partie constante d'une chaîne normale, à l'exception de quelques substitutions ou mutations dans certaines protéines. En ce qui concerne l'extrémité amino-terminale, les constatations sont variables d'une protéine à l'autre : (a) pour certaines protéines, comme deux protéines de $M C L \propto$ récemment étudiées dans notre laboratoire, on ne trouve aucun résidu avant le début de la séquence normale dans un domaine de la région constante. Il est proba- ble (et parfois démontré) qu'en pareil cas, une dégradation protéolytique de l'extrémité amino-terminale de la protéine est survenue après sa synthèse; $(b)$ pour d'autres protéines de MCL (en particulier de la classe $\gamma$ ), la séquence amino-terminale, de longueur variable, est celle d'un $V_{H}$ normal mais incomplet. Il existe alors une véritable délétion interne; $(c)$ dans certaines protéines, on trouve une séquence amino-terminale ne correspondant à aucune séquence de région variable et dont l'origine est totalement inconnue. Cette séquence est tantôt brève (comme dans la protéine de MCL $\propto$ DEF [7]), tantôt longue (comme dans la protéine de MCL $\mu$ BOT [8]). Ces séquences amino-terminales pourraient représenter la traduction d'un segment génomique résultant d'un réarrangement aberrant ou d'une insertion (peut-être d'un oncogène). Enfin, les protéines de MCL $\alpha$ ont une teneur élevée en sucres et certaines protéines de MCL $\gamma$ ou $\mu$ contiennent de la galactosamine qui n'est pas présente dans les chaînes lourdes normales de

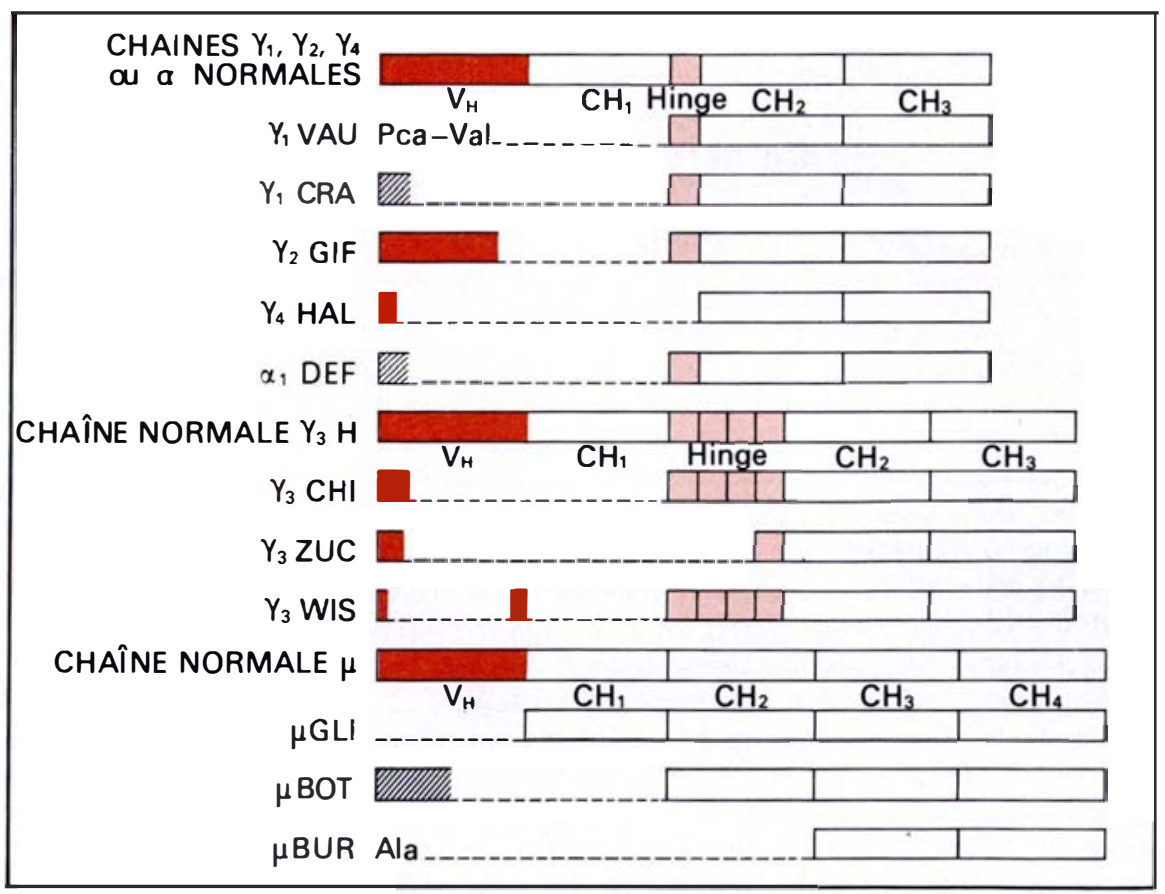

Figure 2. Représentation schématique de la structure de certaines protéines de maladie des chaînes lourdes, comparées à des chaînes lourdes normales de la même classe ou sous-classe. Les barres rouges représentent les régions $V_{H}$, les barres roses la région charnière et les barres hachurées les séquences amino-terminales d'origine inconnue. Les délétions sont représentées par une ligne en pointillés. D'après [4]. 
la classe correspondante.

Seule l'étude des acides nucléiques des cellules lymphoides produisant les protéines de MCL $\alpha$ permettra de comprendre les mécanismes aboutissant aux anomalies de structure résumées ci-dessus. Ces études de biologie moléculaire pourraient en outre permettre de faire progresser nos connaissances sur les mécanismes génétiques normaux.

\section{Premières études} de biologie moléculaire

Dans les deux cas de MCL $\alpha$ qui ont été étudiés par A. Tsapis et M. Bentaboulet dans notre laboratoire, l'ARN messager codant pour la protéine de MCL est plus court qu'un ARNm $\alpha_{1}$ normal. Sa taille $(1,15 \mathrm{~kb}$ dans un cas et $0,95 \mathrm{~kb}$ dans l'autre) est compatible avec une délétion portant sur deux domaines. Ces données excluent les hypothèses d'une simple anomalie de traduction ou d'un simple phénomène de dégradation post-synthétique. En revanche, l'hypothèse d'une protéolyse limitée de l'extrémité N-terminale d'une chaîne $\alpha 1$, porteuse d'une délétion interne, est compatible avec le fait que l'ADNc, préparé à partir de l'ARNm de l'un de ces patients, possède 200 nucléotides en $5^{\prime}$ de la séquence codant pour la région charnière. Ces mêmes constatations ont été faites dans un cas de MCL $\gamma 3$ [9].

L'étude par Southern blot* de l'ADN génomique des deux malades et l'établissement, dans un des cas, de la carte de restriction d'un fragment $\alpha_{1}$ réarrangé, qui a été cloné, ont montré l'existence d'une délétion génomique portant au moins sur une partie de l'exon $\mathrm{CH}_{1}$, l'intron adjacent en 5 ' et la région switch, alors que la région $\mathrm{JH}$ apparaît présente. $\mathrm{La}$ séquence de ce fragment réarrangé, qui est en cours, est nécessaire pour préciser la localisation et l'étendue de la délétion génomique et pour mettre en évidence d'éventuelles anomalies

* Après digestion de PADN par des enzymes de restriction et séparation électrophorétique, les fragments d'ADN sont transférés sur filtre. Le filtre est ensuite hybridé avec une "sonde "radioactive (séquence d'ADN homologue) qui reconnaît les fragments spécifiques. des signaux d'épissage. L'éventuelle insertion d'un oncogène devra bien entendu être recherchée avec soin. Signalons à ce propos que l'étude caryotypique effectuée par $\mathrm{R}$. Berger a pu mettre en évidence chez l'un des malades une translocation 9-14 et chez l'autre une translocation 2-14. Une délétion génomique impliquant l'exon $\mathrm{CH}_{1}$, a été également démontrée dans un cas de MCL $\gamma[10]$. Par contre, dans un cas de MCL $\mu$, il paraît exister seulement une anomalie de transcription [1 11$]$.

Quant à l'absence de chaînes légères, autre anomalie essentielle des protéines de MCL $\alpha$, les études effectuées au niveau cellulaire ont montré qu'il s'agissait en général d'une absence de production [12].

Or, dans les deux cas étudiés dans notre laboratoire, il existe au niveau génomique un réarrangement des séquences $\kappa$ et il y a présence d'un ARNm $\kappa$ non traduit. Une constatation analogue a été effectuée sur un mutant murin, produisant une protéine voisine de celles des MCL humaines [13].

Ces résultats sont préliminaires et ne portent actuellement que sur deux malades. Compte tenu des données établies pour quelques variants de myélomes murins (produisant des chaînes lourdes dont la délétion ne porte en général que sur un seul domaine), on peut s'attendre à ce que les résultats des études de biologie moléculaire varient d'un malade à l'autre. C'est ainsi que deux mutants produisant des châ̂nes $\alpha$ avec une délétion du $\mathrm{CH}_{1}$ de longueur analogue ont des anomalies différentes de l'ADN. L'un a une délétion génomique comprenant l'exon $\mathrm{CH}_{1}$ et une partie des introns adjacents; l'autre a une plus petite délétion qui ne porte pas sur l'intégralité de l'exon $\mathrm{CH}_{1}$, ce qui suggère que les séquences résiduelles de cet exon disparaissent au cours de l'épissage de l'ARN [14].

\section{Expression clinico-pathologique}

La MCL $\propto$ touche le système de l'IgA sécrétoire. Dans trois observations, la prolifération paraît localisée à l'appareil respiratoire mais les données anatomiques sont très incomplètes. Il nous paraît très probable que des formes touchant l'arbre bronchique, les glandes salivaires et d'autres sites de production de l'IgA sécrétoire seront décrites dans l'avenir. Dans la presque totalité des centaines de cas actuellement connus, il s'agit de formes digestives de la maladie [15], avec une prolifération localisée de façon prédominante à l'intestin grêle (dont l'atteinte est habituellement totale, sans intervalle de muqueuse saine) et aux ganglions mésentériques. On observe parfois également une atteinte des muqueuses gastrique, colique et rectale. L'atteinte des ganglions rétropéritonéaux ou médiastinaux, du foie ou de la rate est exceptionnelle, ne survenant qu'à un stade tardif de la maladie.

La MCL $\alpha$ évolue en 2 stades. Le premier est défini par une infiltration lymphoplasmocytaire, non invasive, de la lamina propria de l'intestin grêle et des ganglions mésentériques. L'expression clinique habituelle est celle d'un syndrome de malabsorption ainsi que d'une entéropathie exsudative. Le deuxième stade, souvent précédé de l'apparition, au sein de l'infiltrat lymphoplasmocytaire, de plasmocytes dystrophiques et de cellules de type immunoblastique [16] est un lymphome B, le plus souvent immunoblastique, formant certaines tumeurs circonscrites ou infiltrant de façon extensive les organes atteints. C'est à ce stade que l'on peut observer un syndrome tumoral avec masses abdominales, obstruction chronique du grêle, parfois perforation ou invagination intestinale aiguë. Le sarcome immunoblastique peut être constaté quelques mois après la survenue des premiers symptômes digestifs; à l'inverse, on peut encore se trouver au stade d'une infiltration lymphoplasmocytaire dix ans après le début apparent de la maladie. Les cellules sarcomateuses du second stade dérivent du même clone de cellules $B$ que les cellules plasmocytaires du stade initial [12]. On trouve en effet sur la membrane de ces cellules sarcomateuses des chaînes $\alpha$ sans chaînes légères et les études de biosynthèse ont montré que ces cellules produisent, sans les sécréter, des chaînes $\alpha$ incomplètes. 


\begin{tabular}{|c|c|c|c|}
\hline \multicolumn{4}{|c|}{$\begin{array}{l}\text { ORIGINE GEOGRAPHIQUE DES } 130 \text { PREMIERS PATIENTS } \\
\text { ATTEINTS DE MALADIE DES CHAIINES ALPHA }(a)\end{array}$} \\
\hline 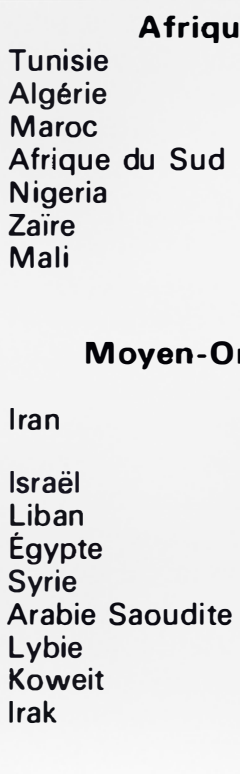 & $\begin{array}{r}13 \\
10 \\
10 \\
2 \\
2 \\
1 \\
1 \\
1 \\
1 \\
1\end{array}$ & \begin{tabular}{l}
$\quad$ Extrême- \\
Pakistan \\
Cambodge \\
Inde \\
Espagne \\
Turquie \\
Italie du Sud \\
Yougoslavie \\
Grèce \\
Finlande \\
Portugal \\
Pays-Bas \\
Grande-Bretagne \\
\multicolumn{1}{c}{ Améric } \\
États-Unis \\
Colombie \\
Mexique \\
Argentine
\end{tabular} & $\begin{array}{r}10 \\
9 \\
6 \\
2 \\
2 \\
1 \\
2 \\
1(b) \\
1(b) \\
\\
1(b) \\
1 \\
1 \\
1\end{array}$ \\
\hline
\end{tabular}

(a) D'après /6/. (b) Forme respiratoire de la maladie.

Au premier stade, et à ce stade seulement, on a pu obtenir chez quelques malades une rémission complète clinique, histologique et immunologique de longue durée après un traitement comportant exclusivement une antibiothérapie par voie orale, en général par les tétracyclines. Cette importante constatation pose le problème de la nature véritablement maligne de l'infiltration lymphoplasmocytaire à ce premier stade. Elle doit être rapprochée des données épidémiologiques qui suggèrent l'intervention de micro-organismes intestinaux dans la genèse de la maladie.

$\mathrm{La}$ forme digestive de la MCL $\alpha$ qui atteint le plus souvent des sujets âgés de 10 à 30 ans, s'observe chez des malades originaires de certaines régions ou y ayant vécu (tableau I) : bassin méditerranéen et en particulier Afrique du Nord, Proche-Orient; plus rarement à notre connaissance, Afrique Noire, Afrique du Sud, Extrême-Orient, Amérique Centrale ou Amérique du Sud. Le seul dénominateur commun à ces patients d'origine ethni- que très variée et d'un niveau socio-économique souvent bas paraît être le haut degré d'infestation intestinale par des microorganismes. Ces données épidémiologiques suggèrent fortement une stimulation antigénique locale intense et prolongée qui pourrait jouer un rôle majeur, prédisposant ou déclenchant, dans la survenue de la prolifération clonale de cellules B. Les études parasitologiques, bactériologiques et virologiques, entreprises dans un nombre limité de cas, n'ont pas mis en évidence d'agent infectieux spécifique. Le rôle probable de ces facteurs d'environnement n'exclut pas la possibilité d'une prédisposition génétique, dont l'existence reste cependant à démontrer. Il est remarquable que la prolifération plasmocytaire, possiblement induite par un ou des micro-organismes intestinaux, aboutisse à la synthèse de protéines de MCL plutôt qu'à celle d'IgA monoclonales complètes qui ne sont que très exceptionnellement observées dans le tableau clinico-pathologique particulier de la MCL $\alpha$

\section{Summary}

Alpha chain disease is the most frequent of the heavy chain diseases. The most reliable method for its diagnosis is immunoelectrophoresis combined to immunoselection. The immunoglobulin molecules produced are deleted $\alpha_{1}$ chains devoid of light chains. The structural defect is usually a deletion involving all or part of the $V_{H}$ region and the first domain of the constant region, the normal sequence starting at the beginning of the hinge region at position 222 . Preliminary results obtained in two patients indicate that the mRNA coding for these abnormal $\alpha_{1}$ chains is shorter than that coding for normal $\alpha_{1}$ chains. Southern blot analysis and the restriction map of a cloned rearranged $\alpha_{1}$ fragment suggest the occurence of a genomic deletion involving at least part of the $\alpha_{1} \mathrm{CH}_{1}$ exon, its $5^{\prime}$ flanking region and the switch region. Although light chains are not produced, the presence of an untranslated $\mathrm{kmRNA}$ was found. Alpha chain disease involves primarily the secretory Ig A system. Most patients are affected with the digestive form of the disease that occurs mainly in the second and third decade of life, with a peculiar geographic distribution. The lymphoid proliferation usually involves the small intestine and mesenteric nodes. The lesions progress from an initial stage of diffuse lymphoplasmacytic infiltration, that can be sometimes cured by oral antibiotics alone, to an overt malignant lymphoma. Environmental factors, providing a protracted antigenic stimulation in the gut, play a triggering or predisposing role. Alpha chain disease is an important model for the understanding of the pathogenesis of human lymphomas.

\section{TIRES A PART}

M. Seligmann : Inscrm U 1 08, hôpital SaintLouis, 75475 Paris Cedex 10. 